\title{
West syndrome caused by homozygous variant in the evolutionary conserved gene encoding the mitochondrial elongation factor GUF1
}

\author{
Ali Abdullah Alfaiz ${ }^{1,2,3,11}$, Verena Müller ${ }^{4,11}$, Nadia Boutry-Kryza ${ }^{5,6,7,11}$, Dorothée Ville ${ }^{8}$, Nicolas Guex ${ }^{1,2}$, \\ Julitta de Bellescize ${ }^{9}$, Clotilde Rivier ${ }^{10}$, Audrey Labalme ${ }^{5}$, Vincent des Portes ${ }^{6,8}$, Patrick Edery ${ }^{5,6,7}$, \\ Marianne Till ${ }^{5}$, Ioannis Xenarios ${ }^{1,2}$, Damien Sanlaville ${ }^{5,6,7}$, Johannes M Herrmann ${ }^{4}$, Gaétan Lesca ${ }^{\star 5,5,7,12}$ \\ and Alexandre Reymond ${ }^{\star, 1,12}$
}

\begin{abstract}
West syndrome (WS), defined by the triad of infantile spasms, pathognomonic hypsarrhythmia and developmental regression, is a rare epileptic disease affecting about 1:3500 live births. To get better insights on the genetic of this pathology, we exomesequenced the members of a consanguineous family affected with isolated WS. We identified a homozygous variant (c.1825G > T/p.(Ala609Ser)) in the GUF1 gene in the three affected siblings. GUF1 encodes a protein essential in conditions that counteract faithful protein synthesis: it is able to remobilize stuck ribosomes and transiently inhibit the elongation process to optimize protein synthesis. The variant identified in the WS family changes an alanine residue conserved in all eukaryotic organisms and positioned within the tRNA-binding moiety of this nuclear genome-encoded mitochondrial translational elongation factor. Yeast complementation assays show that the activity of GUF1 ${ }^{A 609 S}$ is modified in suboptimal environments. We suggest a new link between improper assembly of respiratory chain complexes and WS.
\end{abstract}

European Journal of Human Genetics (2016) 24, 1001-1008; doi:10.1038/ejhg.2015.227; published online 21 October 2015

\section{INTRODUCTION}

Infantile spasms (IS) are an age-dependant epileptic syndrome characterized by clinical spasms occurring in clusters and abnormal pattern on electroencephalography (EEG). ${ }^{1,2}$ Their incidence ranges from 2 to $5 / 10000$ per live births. ${ }^{3}$ West syndrome (WS) is one of the well-defined epileptic syndromes. It associates IS with a typical EEG pattern called hypsarrhythmia consisting of random, high-voltage, nonsynchronous spikes and slow waves and developmental delay. Despite improvements of early diagnosis and appropriate treatment, prognosis of IS/WS remains poor; 75-90\% of patients show cognitive impairment, while $50-60 \%$ of affected children have recurrent seizures at 5 years of age. ${ }^{4,5}$ The etiology of IS/WS is highly heterogeneous. ${ }^{6}$ They occur as isolated feature or as part of more complex disease syndrome. They typically are the consequence of acquired events, such as hypoxemia or intracranial hemorrhage, or determined by genetic factors. The most frequent genetic causes of IS are mutations in TSC1 (about 9\% of all IS/WS patients, OMIM\#191100), CDKL5, ARX and STXBP1 genes, as well as multiple genomic imbalances, the commonest being Pallister-Killian syndrome (tetrasomy 12p; OMIM\#601803) and 1 p36 deletion (OMIM\#607872). ${ }^{7-13}$ These and other genetic causes such as genes encoding proteins involved in inborn errors of metabolism were recently reviewed in Paciorkowski et al. ${ }^{14}$ IS/WS also occur with a lower prevalence as a feature of other genetic diseases, such as Down syndrome. ${ }^{14}$ This heterogeneity often impedes the identification of the etiology of IS/WS within the clinical practice. ${ }^{15}$ Despite early clinical diagnosis and appropriate treatment, prognosis remains a major concern with frequent intellectual deficiency (75-90\%) and recurrence of seizures later in life (50-60\%). ${ }^{16}$ Definite etiological diagnosis can give information about the prognosis and is mandatory for accurate genetic counseling. Recent studies based on whole-exome sequencing identified de novo genetic alterations in GABRB3, CACNA1A, CHD2, FLNA, GABRA1, GRIN1, GRIN2B, HNRNPU, IQSEC2, MTOR, NEDD4L, STXBP1, CASK and ALG13 and recessive variants in $P N P O$ and $A D S L .{ }^{17,18}$ These studies suggested that many other genes underlying IS/WS remain to be found.

We report on a consanguineous family with three siblings (a female and two males) affected by WS and severe neurological impairment. They had no malformations or other clinical features, and both biochemical screening and array comparative genomic hybridization results were normal. The family structure prompted us to use wholeexome sequencing to find the causative gene.

\footnotetext{
${ }^{1}$ Center for Integrative Genomics, University of Lausanne, Lausanne, Switzerland; ${ }^{2}$ Swiss Institute of Bioinformatics (SIB), Lausanne, Switzerland; ${ }^{3}$ King Abdullah International Medical Research Center (KAIMRC), King Saud Bin Abdulaziz University for Health Sciences (KSAU-HS), Riyadh, Saudi Arabia; ${ }^{4}$ Division of Cell Biology, Department of Biology, University of Kaiserslautern, Kaiserslautern, Germany; ${ }^{5}$ Department of Constitutional Cytogenetics, Lyon Hospices Civils, Lyon, France; ${ }^{6}$ Claude Bernard Lyon I University, Lyon, France; ${ }^{7}$ CRNL, CNRS UMR 5292, INSERM U1028, Lyon, France; ${ }^{8}$ Center of Reference for Tuberous Sclerosis and Rare Epileptic Disorders, Hospices Civils de Lyon, Lyon, France; ${ }^{9}$ Department of Pediatric Epileptology, Lyon Hospices Civils, Lyon, France; ${ }^{10}$ Department of Pediatrics, Hopital Nord-Ouest, Villefranche-sur-Saône, France *Correspondence: Dr G Lesca, Service de Cytogénétique Constitutionnelle, Groupement Hospitalier Est, 59 Boulevard Pinel, Bron Cedex, Lyon 69677, France. Tel: +33 4278555 73; Fax: +33 4721297 10; E-mail: gaetan.lesca@chu-lyon.fr

or Professor A Reymond, Center for Integrative Genomics, University of Lausanne, Genopode Building, Lausanne 1015 , Switzerland. Tel: +41 21 692 3960 ; Fax: +41 21692 3965; E-mail: alexandre.reymond@unil.ch

${ }^{11}$ These authors contributed equally to this work.

${ }^{12}$ These senior authors contributed equally to this work.

Received 20 January 2015; revised 12 September 2015; accepted 18 September 2015; published online 21 October 2015
} 


\section{MATERIALS AND METHODS}

\section{Detailed patients' description}

Familial history. There was a family history of death, in the first week of life of a paternal aunt and uncle from unknown causes, and of epilepsy without developmental delay in a different paternal aunt and a paternal cousin.

Patient IV-1. This boy was born at the thirty-sixth week of gestation after an uneventful pregnancy, with weight $2670 \mathrm{~g}(-1.5 \mathrm{SD})$, length $42 \mathrm{~cm}(-3 \mathrm{SD})$ and occipital frontal circumference $(\mathrm{OFC}) 36 \mathrm{~cm}(+2 \mathrm{SD})$. He had hyaline membrane disease with favorable outcome but had feeding difficulties and periods of lethargy. EEG and transcranial sonography were normal at birth. At 5 months of age, systematic pediatric examination disclosed severe hypotonia with poor eye contact that had not been noticed by the parents. EEG showed hypsarrhythmia and electroclinical spasms were video-recorded (Supplementary Figure S1 and S2). He had no dysmorphic features. Multiple simultaneous or successive antiepileptic drug attempts (Vigabatrin, Prednisolone, Tetracosactide, Nitrazepam, Phenytoine, Topiramate, Levetiracetam, Lamotrigine, Oxcarbamazepine, Rufinamide), as well as Pyridoxal and Pyridoxal phosphate, were unsuccessful, and evolution was marked by persistence of spasms and myoclonia. Neurological outcome was also poor. At last followup, at 6 years of age, he had profound psychomotor delay with no babbling, severe axial hypotonia with spastic tetraparesis, no ability to handle or grasp objects and he displayed some dystonic fits and choreoathetotic movements. Eye contact was partially preserved.

Brain MRI performed at epilepsy onset was normal, but diffuse cortical atrophy was present at 2 years of age (Supplementary Figure S3). Electromyography and fundoscopy did not reveal any anomaly. Blood karyotype did not show any chromosomal imbalance. Metabolic dosages in blood included: ammonemia, lactate, pyruvate, amino-acid chromatography, organic acid chromatography, pipecolic acid, SAICAR (succinyl-5-aminoimidazole-4-carboxamide-1-ribose-5'-phosphate), testing for congenital disorders of glycosylation, dosages of beta-glycosydase (GM1) and beta-hexosaminidase (GM2) in leucocytes, dosages of palmitoyl thio-esterase- 1 and tripeptidyl peptidase-1 in leukocytes were within normal range. In the CSF, glucose, lactate $(1050 \mu \mathrm{mol} / \mathrm{l})$ and pyruvate $(67 \mu \mathrm{mol} / \mathrm{l})$ dosages were normal, whereas dosages of neurotransmitter metabolite analysis showed a slight decreased level of homo vanillic acid, which was considered non-significant (HVA: $258 \mathrm{nmol} / \mathrm{l}$ (normal range: 429-789 nmol/l), MHPG: $54 \mathrm{nmol} / \mathrm{l}$ (normal range: 33-71 nmol/l), HVA/ MHPG ratio: 4.8 (normal range: 5-15)). No variants were found in the CDKL5 and ARX genes.

Patient IV-2. Patient IV-1's sister had intrauterine growth retardation. She was born at the thirty-fifth week of gestation by cesarean section after an uneventful pregnancy, with weight $1400 \mathrm{~g}(<-3 \mathrm{SD})$, length $44 \mathrm{~cm}(-2 \mathrm{SD})$, and OFC $29.5 \mathrm{~cm}(-2.5 \mathrm{SD})$. The first days of life were characterized by hyaline membrane disease with favorable outcome. Neonatal EEG was performed because of the early onset of epilepsy in the previous child; cranial ultrasonography and fundoscopy were normal. Careful pediatric follow-up was performed and slight hypotonia with good eye contact was noticed before the first epileptic manifestations. Spasms onset started at 5 months of life. EEG showed hypsarrhythmia, and electroclinical spasms were recorded (Supplementary Figure S1). Epilepsy onset was associated with psychomotor arrest. At examination, she displayed axial hypotonia, alteration of eye contact and no dysmorphic features. Brain MRI was normal. Lymphocyte karyotype, mutation screening of the ARX gene, CSF lactate and pyruvate dosages were normal (lactate: $1110 \mu \mathrm{mol} / \mathrm{l}$, pyruvate: $85 \mu \mathrm{mol} / \mathrm{l}$, lactate/pyruvate ratio: 13 ), and CSF neurotransmitter metabolite analysis showed slight decreased level of vanillic acid, which was considered non-significant (HVA: $283 \mathrm{nmol} / \mathrm{l}$ (normal range: $295-932 \mathrm{nmol} / \mathrm{l}$ ), MHPG: $51 \mathrm{nmol} / \mathrm{l}$ (normal range: $4-50 \mathrm{nmol} / \mathrm{l}$ ), HVA/ MHPG ratio: 1.2 (normal range: 1.11-3.48)). No antiepileptic treatment (Vigabatrin, Clonazepam, Prednisolone and Levetiracetam) was effective. Neurological outcome was very poor. She died at 1 year of age from SUDEP (sudden unexpected death in epilepsy) during a H1N1 virus and respiratory syncytial virus co-infection.

Patient IV-3. Patient IV-1's younger brother was born at the thirty-seventh week of gestation after an uneventful pregnancy, with weight $3350 \mathrm{~g}$ (mean), length $50 \mathrm{~cm}(+1 \mathrm{SD})$ and $\mathrm{OFC} 35.5 \mathrm{~cm}(+1 \mathrm{SD})$. Hypotonia and psychomotor delay had been noticed since the first days of life with preserved eyes contact. Neonatal transcranial sonography and EEG were normal. First spasms occurred when he was 4 months with psychomotor delay. EEG showed hypsarrythmia and electroclinical spasms were recorded (Supplementary Figure S1). He had no dysmorphic feature. Epilepsy could not be controlled despite treatment with Vigabatrin, and neurological outcome was poor. At last follow-up, he was 15 months of age, spasms and myoclona occurred several times per day. He displayed severe hypotonia with slight spasticity, fluctuating eye contact, no babbling and no ability to handle or grasp objects. No dystonic fit was noticed until now. Lymphocyte karyotype, array CGH, blood amino-acid chromatography and CSF lactate and pyruvate dosages (lactate: $1090 \mu \mathrm{mol} / \mathrm{l}$, pyruvate: $101 \mu \mathrm{mol} / \mathrm{l}$, lactate/pyruvate ratio: 11). CSF neurotransmitter metabolite analysis showed normal values: HVA $528 \mathrm{nmol} / \mathrm{l}$ (normal range: $310-1328$ nmol/l), MHPG $66 \mathrm{nmol} / \mathrm{l}$ (normal range: $30-168 \mathrm{nmol} / \mathrm{l})$, HVA/MHPG ratio: 8 (normal range: $5-15$ ). No brain MRI was performed.

\section{Exome sequencing and analysis}

All samples used in this study were collected with appropriate informed consent and approval of the local ethics committees. We performed exome sequencing on both parents and the three affected siblings. Exomes were captured using the Agilent Sureselect Human All Exon V4 Enrichment Kit (Santa Clara, CA, USA) and multiplex sequenced on two Illumina Hiseq lanes (San Diego, CA, USA). Sequencing reads were aligned to the reference human genome using BWA, ${ }^{19}$ and variants were called with GATK $^{20}$ using a Minimum Confidence Calling and Emitting score of 30, Base Quality Score of 17 and Mapping Quality score of 20. Prior to variant calling, each sample was screened for duplicates using PICARD tools (http://picard.sourceforge.net), base quality score recalibration and InDel realignment with GATK. ${ }^{21}$ We reached a mean bait coverage of 87 fold, with $76 \%$ of the nucleotides with at least a 20 -fold coverage on average. We filtered out synonymous variant, as well as the variants previously recorded in $\mathrm{dbSNP}$, the 1000 genome project, the HapMap projects and our in-house exome variant server ( $>1000$ exomes performed) with $\mathrm{MAF}>0.1 \%$. The remaining variants were sorted according to their inheritance pattern with VCFtools $^{22}$ and Snpeff (http://snpeff.sourceforge.net/). The c.1825G $>$ T, p. (Ala609Ser) variant was submitted to the GUF1 locus-specific database of the Leiden Open Variation Database (www.lovd.nl/GUF1). Of note, the Exome Aggregation Consortium (ExAC), Cambridge, MA, USA (http://exac.broadinstitute.org) (accessed March 2015) uncovered 288 missense or loss-offunction variants in GUF1 of which only chr4:44691873 G > C p.(Gly406Arg) was (i) deleterious according to SIFT or Polyphen; (ii) not found in homozygosity; and (iii) evolutionary constrained. We acknowledge that we could, however, not exclude cases of compound heterozygosity.

\section{Yeast complementation}

We used cDNA from a WS patient and from a control as template to amplify the coding sequences of the normal and variant human GUF1, hGUF1 and hGUF1 $^{\mathrm{A} 609 \mathrm{~S}}$, respectively. These sequences were cloned into the pYX232 yeast expression plasmid (Novagen, EMD Millipore, Billerica, MA, USA) under control of a constitutive triosephosphate isomerase promoter. These plasmids were transformed into $\Delta g u f 1$ yeast strains. The resulting strains were grown to logarithmic growth phase, and serial dilutions were spotted onto agar plates containing $0.5 \%$ glycerol. Upon incubation at elevated growth temperatures $\left(37^{\circ} \mathrm{C}\right)$, the $\Delta$ gufl strains showed a mild growth defect confirming earlier studies. ${ }^{23}$ The growth defect was further aggravated by overexpression of either hGUF1 or hGUF1 ${ }^{\mathrm{A} 609 \mathrm{~S}}$, indicating that both variants interfere with mitochondrial translation machinery with similar degrees. Next we evaluated whether the expression of the human Gufl proteins influences mitochondrial protein expression. We isolated mitochondria by subcellular fractionation of control, $\Delta g u f 1$ and $\Delta g u f 1$-overexpressing hGUF1 or hGUF1 ${ }^{\mathrm{A} 609 \mathrm{~S}}$ cells and analyzed the levels of the mitochondrially encoded protein Cox2. As Cox2 is a central part of cytochrome $c$ oxidase (Complex IV), a decrease of its level would indicate a severe defect in respiration. We found, however, no changes in the levels of the Cox 2 proteins. To assess mitochondrial protein synthesis more directly, we incubated mitochondria in the presence of ATP, amino acids and radiolabeled $\left[{ }^{35} \mathrm{~S}\right]$-methionine for $10 \mathrm{~min}$ at either 15 or $30^{\circ} \mathrm{C}$. Mitochondria were then 
reisolated, and newly synthesized translation products were visualized by autoradiography as described in Bauerschmitt et al. ${ }^{23}$ Again, no differences were observed between $\Delta g u f 1$ and $\Delta g u f 1$-overexpressing hGUF1 or hGUF1 ${ }^{\mathrm{A} 609 \mathrm{~S}}$ cells, all strains showing a similar translation pattern. Whereas deletion of GUF1 leads to almost no detectable defects in yeast, Gufl activity is critical in the oxal $\Delta C$ mutant. ${ }^{23}$ This strain lacks part of the C-terminal ribosomebinding domain of the mitochondrial Oxal insertase (residues 332-402). It shows no obvious defect in the biogenesis of mitochondrial translation products $^{24,25}$ but exhibits a strong synthetic growth defect with $\Delta g u f 1$ at high temperatures and on non-fermentable carbon sources. We used this synthetic defect to analyze the activity of the human Gufl variant protein in yeast mitochondria. To this end, we transformed the oxal $\Delta C \Delta$ gufl double mutant with plasmids expressing either hGufl or hGufl ${ }^{\mathrm{A} 609 \mathrm{~S}}$ and tested the growth of the resulting strains in a drop dilution assay. The strong synthetic effect observed with the oxal $\Delta C \Delta g u f 1$ double mutant at higher temperatures was completely suppressed upon expression of both hGufl and hGufl ${ }^{\mathrm{A} 609 \mathrm{~S}}$. This shows that both variant proteins, when expressed in yeast, are targeted to mitochondria where they are able to take over the function of yeast Gufl. To eventually detect subtle differences between hGufl or hGufl ${ }^{\mathrm{A} 609 \mathrm{~S}}$ in the above complementation assays, we compared the fitness of oxal $1 \mathrm{C} \Delta \mathrm{guf} 1+\mathrm{hGufl}$ and oxa1 $1 \mathrm{C} \Delta$ guf $1+\mathrm{hGuf1}{ }^{\mathrm{A} 609 \mathrm{~S}}$ in a competition assay. Both strains were grown in the same culture over a 12-day period while the proportion of each mutant was assessed. To discriminate both strains, we transformed the oxal $\Delta C \Delta g u f 1$ +hGufl cells with a plasmid harboring a uracil marker and the oxalsC $\Delta g u f 1$ + hGufl ${ }^{\mathrm{A} 609 \mathrm{~S}}$ mutant with a plasmid containing a leucine marker. Both mutants were pregrown to log phase in glycerol-containing medium and inoculated at equal proportions into the common culture. The culture was repeatedly diluted to promote continuous cell growth. Aliquots were taken after 0, 3, 6, 9 and 12 days, and the proportion of each mutants was determined by plating different volumes on uracil- or leucine-deficient plates. Even after prolonged growth (equivalent to about 70 generations) and using an increased granularity of the measurement, the proportion of both strains remained equal, indicating that both mutants grew with identical rates. We then tested complementation of oxa $1 \Delta C \Delta g u f 1$ by the two variants in suboptimal environments, that is, $0.5 \%$ glycerol plates supplemented with potassium tellurite, magnesium or chloramphenicol (see main text for details).

\section{RESULTS}

We describe the characterization of a consanguineous pedigree with three siblings affected with WS suggestive of a recessive monogenic disorder (Figure 1a). The parents were healthy first cousins from Algerian origin. There was no previous family history of severe

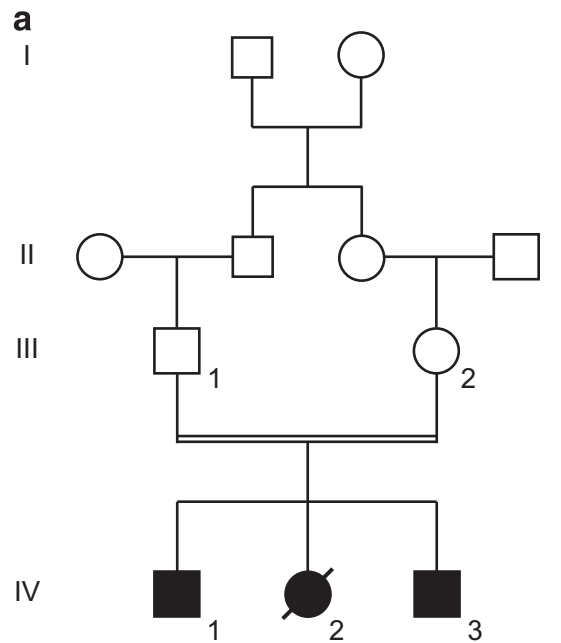

epileptic or neurocognitive disorder. The phenotypes of the three siblings (and relatives) are summarized in Table 1. In summary, the three siblings had normal EEG and cranial ultrasonography at birth. Hypotonia preceded the first spasms, which set in at 5, 5 and 4 months of age in patient IV-1, IV-2 and IV-3, respectively. EEG at 5 months showed hypsarrhythmia, as well as electroclinical spasms in the three siblings (Supplementary Figures S1 and S2). Epilepsy onset was associated with psychomotor arrest and poor neurological outcome. Antiepileptic drug treatments, sometimes simultaneous or successive, were inefficient (Table 1). Levels of blood metabolites, as well as CSF lactate and pyruvate concentrations, were normal. We recorded, however, a slight decrease in homovanillic acid levels in patients IV-1 and IV-2. Brain MRI of patient IV-1 revealed a diffuse cortical atrophy (Supplementary Figure S3 and Table 1). Of note, patient IV-2 died at 1 year of age from SUDEP during a H1N1 virus and respiratory syncytial virus co-infection.

We performed exome sequencing on both parents and the three affected siblings. After filtering known and synonymous variants and sorting according to inheritance pattern, we found a single unknown variant that segregated with the disease and putatively affected the protein it encodes (SIFT score $=0.05 ;{ }^{26}$ Mutation Taster diseasecausing prediction: $P=1.0$ ). This variant was homozygote in the three siblings and heterozygote in the parents and thus was compatible with an autosomal-recessive inheritance of the disease. We found no variant compatible with a de novo variant in the three affected assuming a possible germline mosaicism of one of the parents. The identified variant is a $\mathrm{G}$ to $\mathrm{T}$ transversion (chromosome 4 at nucleotide 44,697,741 [hg19]) in the 15th exon of the GUF1 gene (GTPase of unknown function 1; r. $(2032 \mathrm{~g}>\mathrm{u})$ (c.1825G $>$ T) in NM_021927.2), which substitutes an alanine into a serine codon at position 609 (p.(Ala609Ser)). We confirmed by Sanger sequencing the heterozygosity and homozygosity of this variant in the parents and siblings, respectively (Figure 1b). GUF1 encodes a universally conserved GTPase in mitochondria and chloroplasts, as well as in prokaryotes where it is named EF4 or LepA. We used an X-ray structure of EF4, a cryo-EM model of EF4 bound to an elongating ribosome with a backtranslocated tRNA and Swiss-PdbViewer ${ }^{27-29}$ to model a threedimensional representation of the human GUF1 bound to a tRNA (Figure 2a). The variant we identified in the WS family substitutes an

b

III1

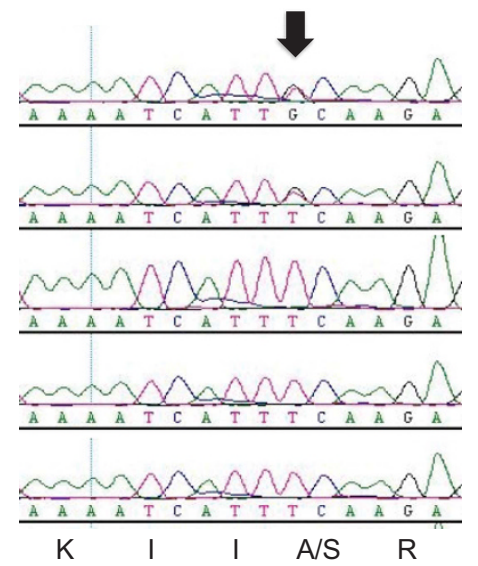

Figure 1 (a) Four generation pedigree of the consanguineous family with three siblings affected with WS. (b) Sequencing fluorograms showing the alanine to serine variant of GUF1 codon 609 (black arrow) in the heterozygote and homozygote state in the two parents and their affected siblings, respectively. Codon translation is indicated at the bottom. 
Table 1 Summary of phenotypic features presented by the three affected siblings

\begin{tabular}{|c|c|c|c|}
\hline Patient & $I V-1$ & $I V-2$ & IV-3 \\
\hline Sex & Male & Female & Male \\
\hline Born after weeks of gestation (weeks) & 36 & $35^{a}$ & 37 \\
\hline Weight (g) & 2670 & 1400 & 3350 \\
\hline Length $(\mathrm{cm})$ & 42 & 44 & 50 \\
\hline OFC (cm) & 36 & 29.5 & 35.5 \\
\hline $\begin{array}{l}\text { Hyaline membrane disease with } \\
\text { favorable outcome }\end{array}$ & Yes & Yes & No \\
\hline Neonatal EEG & Yes & Yes & No \\
\hline Neonatal cranial ultrasonography & Normal & Normal & Normal \\
\hline Spasms onset (months) & 5 & 5 & 4 \\
\hline EEG at 5 months & $\begin{array}{l}\text { Hypsarrhythmia and } \\
\text { electroclinical spasms }\end{array}$ & $\begin{array}{l}\text { Hypsarrhythmia and } \\
\text { electroclinical spasms }\end{array}$ & $\begin{array}{l}\text { Hypsarrhythmia and } \\
\text { electroclinical spasms }\end{array}$ \\
\hline Hypotonia & $\begin{array}{l}\text { No hypotonia during the first months of life } \\
\text { Severe hypotonia with poor eye contact at } \\
5 \text { months and at last follow-up }\end{array}$ & $\begin{array}{l}\text { Slight hypotonia with good eye contact before } \\
\text { first epileptic manifestations } \\
\text { Severe hypotonia at last follow-up }\end{array}$ & $\begin{array}{l}\text { Hypotonia with preserved eye contact } \\
\text { since the first days of life } \\
\text { Severe hypotonia at last follow-up }\end{array}$ \\
\hline Unsuccesful epileptic treatment with & Multiple ${ }^{\mathrm{b}}$ & Multiple ${ }^{c}$ & Vigabratin \\
\hline Brain MRI & $\begin{array}{l}\text { Normal at } 5 \text { months } \\
\text { Major diffuse cortical atrophy at } 2 \text { years }\end{array}$ & Normal at 5 months & Not performed \\
\hline Dysmorphic features & No & No & No \\
\hline Karyotype & Normal & Normal & Normal \\
\hline Array-CGH & Not performed & Not performed & Normal \\
\hline Variants in $A R X$ (Sanger seq) & None & None & Not performed \\
\hline Variants in CDKL5 (Sanger seq) & None & Not performed & Not performed \\
\hline Metabolic dosage in blood & Normal & Normal & Normal \\
\hline Lactate and pyruvate in CSF & Normal & Normal & Normal \\
\hline Homovanillic acid in CSF ( $\mathrm{nmol} / \mathrm{l})$ & 258 & 283 & 528 \\
\hline
\end{tabular}

${ }^{\mathrm{a} B y}$ cesarean section.

bVigabatrin, Prednisolone, Tetracosactide, Nitrazepam, Phenytoine, Topiramate, Levetiracetam, Lamotrigine, Oxcarbamazepine, Rufinamide, as well as Pyridoxal and Pyridoxal phosphate.

cVigabatrin, Clonazepam, Prednisolone and Levetiracetam.

alanine residue of the C-terminal Domain (CTD) that is conserved in all eukaryotes from budding yeast to human and the majority of prokaryotes (A609; an A residue is found at this position in 497 out of 539 EF4/GUF1 alignments; Supplementary Figure S4). The CTD, which is not found in other translational GTPases, ${ }^{30}$ participates in the binding of the tRNA molecule via a beta-sheet ${ }^{28}$ (Figure $2 b$ and $c$ ).

GUF1 is able to back-translocate the tRNA on ribosomes and as increasing amount of the protein resulted in reduced translational error rates, it was suggested that EF4 controls the fidelity of translation. ${ }^{30}$ The extreme conservation across phyla is 'paradoxically associated with an apparent lack of phenotype in deletion mutants' ${ }^{31}$ For example, while E. coli cells lacking the EF4 gene have no ostensible defects in cell growth or protein export under normal medium and temperature, they show defective growth under stress conditions ${ }^{32}$ (see below). Similarly, EF4 is one of the 10 genes essential to the survival of Helicobacter pylori in low $\mathrm{pH}$ environment. ${ }^{33}$ In metazoan, the lack of EF4/GUF1 was associated with mitochondrial dysfunction, in particular disruption of mitochondrial respiratory chain complex IV. ${ }^{34,35}$ These and other results induced Zhang and Qin ${ }^{31}$ to suggest that 'under special ionic stress, for example, extreme $\mathrm{pH}$ environments for bacteria or proton gradient around a mitochondrion, the translation process is much more dependent on EF4'. To assess the functional effect of the c.1825G $>$ T variant, we used a complementation approach (see Materials and Methods). In budding yeasts, GUF1 is important for mitochondrial protein synthesis under suboptimal conditions. ${ }^{23}$ Yeasts that lack the GUF1 $(\Delta g u f 1)$ elongation factor show a mild growth defect under extreme growth-limiting concentrations of non-fermentable carbon sources and low and high temperature. On the contrary, overexpression of Gufl exhibits a pronounced negative effect, ${ }^{23}$ consistent with the observation that overproduction of LepA stalls translation in bacteria. ${ }^{30}$ We found that overexpression of hGUF1 and hGUF1 ${ }^{\mathrm{A} 609 \mathrm{~S}}$ in $\Delta g u f 1$ had comparable effect on growth, mitochondrial protein expression and translation (Supplementary Figure S5A and D and Supplementary Materials). Likewise, hGUF1 and $\mathrm{hGUF1}^{\mathrm{A} 609 \mathrm{~S}}$ were both able to suppress the strong synthetic growth defect of the oxa1 $\Delta C \Delta g u f 1$ double mutant on plates and in direct competition assays (Figure 3, Supplementary Figure S6A and B and Supplementary Materials). Recent results suggest that EF4 is essential in conditions that counteract faithful protein synthesis such as upon exposure to magnesium salts ${ }^{36}$ and potassium tellurite. ${ }^{32}$ In these suboptimal environments, Guf1/LepA activity remobilizes stuck ribosomes and transiently inhibits the elongation process to optimize protein synthesis. ${ }^{36,37}$ We therefore assessed complementation on plates supplemented with potassium tellurite, magnesium or low levels of chloramphenicol. Under these conditions, respiratory growth of the oxal $\Delta C \Delta g u f 1$ double mutant was strictly dependent on the expression of the human Guf1 protein (Figure 3). Interestingly, when compared with hGUF1, expression of hGUF1 ${ }^{\text {A609S }}$ showed an increased resistance to some stress conditions such as exposure to tellurite or high magnesium concentrations combined with growthlimiting concentrations of non-fermentable carbon sources (Figure 3). These results encouraged us to assess mitochondrial protein synthesis in suboptimal environments. The tellurite-sensitive oxalsC $\Delta g u f 1$ double mutant effectively synthesizes mitochondrial proteins both in the presence and absence of tellurite. Whereas overexpression of hGUF1 in the double mutant strain was sensitive to this oxidizing compound, complementation with the hGUF1 ${ }^{\mathrm{A} 609 \mathrm{~S}}$ variant showed an intermediate phenotype (Figure 4). Thus tellurite exposure blocks 
protein synthesis in a GUF1-dependent manner in agreement with the notion that LepA counteracts protein synthesis to prevent the misincorporation of non-cognate amino acids in stressful conditions. ${ }^{36,37}$ The improved growth observed with the p.(A609S) variant suggests that this variant is less proficient than wild-type GUF1 in this stalling and hence possibly in limiting misincorporation.
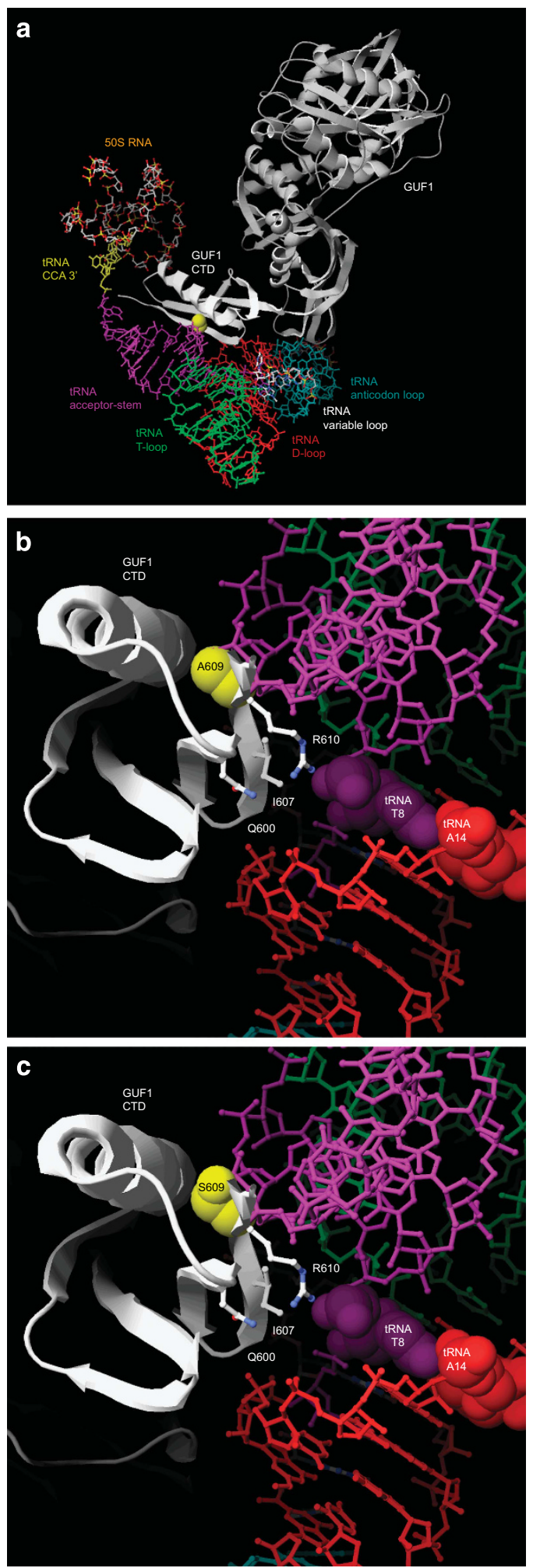

Collectively, these data show that, while the hGuf1 ${ }^{\mathrm{A} 609 \mathrm{~S}}$ variant is still functional, its activity is modified under particular conditions (at least in yeasts).

\section{DISCUSSION}

Epileptic seizures of various types have been reported in one- to two-thirds of patients with mitochondrial chain respiratory disease. ${ }^{38,39}$ These so-called 'mitochondrial epilepsies' are clinically and genetically heterogeneous diseases; disease-causing mutations have been reported in many of the 37 mitochondrially encoded genes and at least 31 nuclear genes (reviewed in $\operatorname{Rahman}^{40}$ ). Mutated genes encode different subunits of the respiratory chain or proteins that have a role in mitochondrial genome maintenance, translation and replication, as well as enzymes involved in the biosynthesis of coenzyme Q10 or the mitochondrial glutamate carrier SLC25A22 (reviewed in $\operatorname{Rahman}^{40}$ ). IS/WS are, however, rare within known

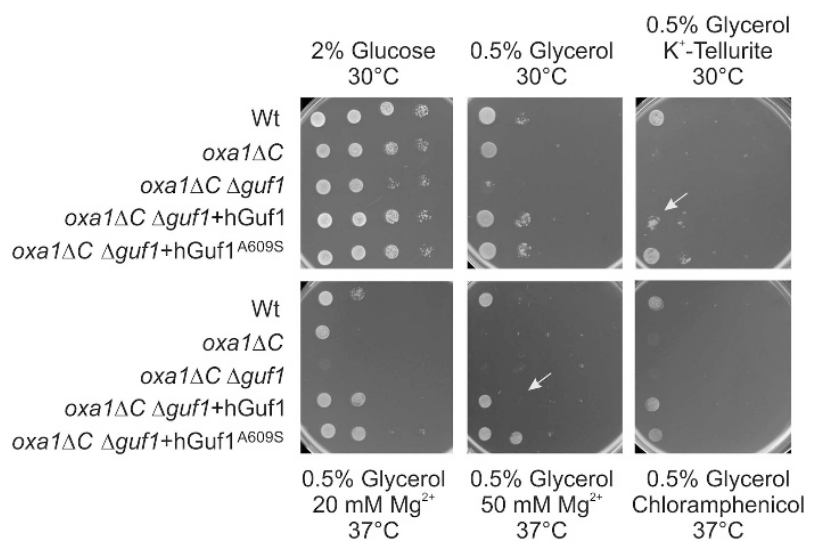

Figure 3 The hGUF1 ${ }^{A 609 S}$ variant exhibits increased activity in some suboptimal environments. The indicated strains were grown in full medium containing galactose to log phase. Serial 10 -fold dilutions were spotted on YP plates containing $2 \%$ glucose, $0.5 \%$ glycerol or $0.5 \%$ glycerol supplemented with potassium tellurite, magnesium or chloramphenicol at the indicated concentrations and plates were incubated at 30 or $37^{\circ} \mathrm{C}$. The decreased ability of hGUF1 to complement the respiratory growth of the oxalsC $\Delta$ guf1 double mutant on a non-fermentable carbon source supplemented with a high concentration of magnesium or with potassium tellurite compared with hGUF1 ${ }^{\mathrm{A} 609 \mathrm{~S}}$ is indicated by arrows.

Figure 2 (a) Three-dimensional representation of the human GUF1 (light gray) and its CTD (white) in contact with a tRNA in the A-site of the ribosome. The different domains of the tRNA are color-coded: CCA 3' (yellow), acceptor stem (purple), T-loop (green), D-loop (red), and anticodon loop (turquoise). The A609 residue is highlighted in yellow. (b) Three-dimensional model of the GUF1 tRNA-binding moiety (white) bound to the tRNA acceptor stem (purple) and D-loop (red). The T8 and A14 nucleotidic positions that are unconstrained in human mitochondrial tRNAs are emphasized (see text for details). The alanine 609 residue (highlighted in yellow) is located on the external beta-strand within the KIIARETV stretch (defined as 'contact 2' in Connell et aR8), close to the evolutionary conserved glutamine (Q600), branched hydrophobic (1607) and Arginine (R610) residues. Their sidechains form a tightly packed motif that might be important for the proper recognition of the tRNA in the region located between the acceptor stem and the D-loop. The T-loop and part of the anticodon loop are indicated in green and turquoise, respectively. (c) The addition of a hydroxyl group at position 609 (p.(A609S) variant; compare with panel $(\mathbf{b})$ ) results in a bulkier residue, which might interfere with the tRNA acceptor stem by modifying the 'contact 2' moiety. For color codes, see panel (b). 

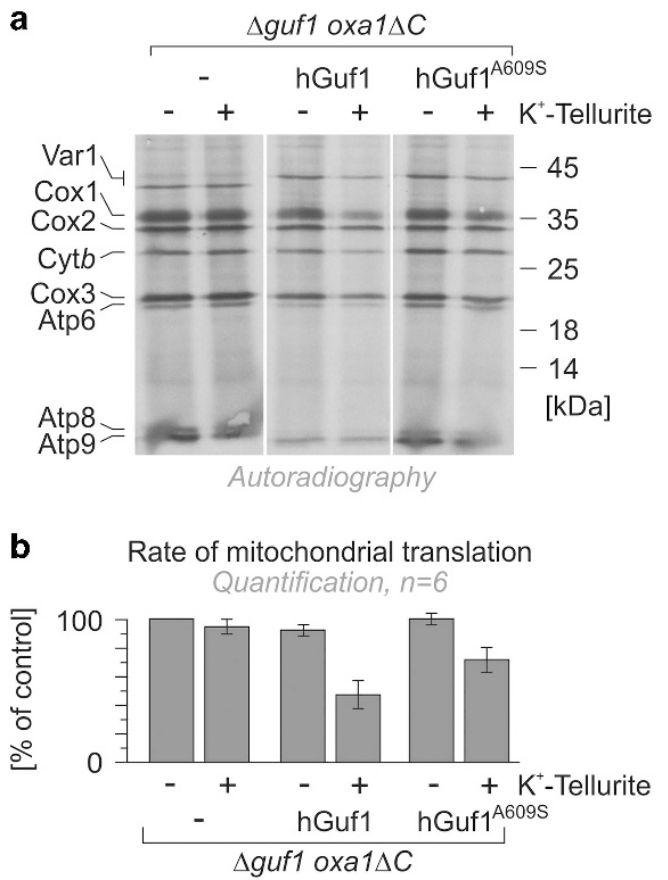

Figure 4 The oxal $\Delta C$ gguf1 double mutant effectively synthesizes mitochondrial proteins in the presence of tellurite, whereas double mutants overexpressing hGUF1 and hGUF1 ${ }^{A 609 S}$ are highly and moderately sensitive to this oxidizing compound, respectively. The strains described in Figure 3 were incubated with cycloheximide to block cytosolic translation, and mitochondria were isolated, incubated in the presence of ATP, amino acids and radiolabeled $\left.{ }^{35} \mathrm{~S}\right]$-methionine before visualization of newly synthesized translation products by autoradiography. Visualized bands are annotated on the left, while molecular weight markers are indicated on the right (a). The rate of mitochondrial translation is quantified in panel (b).

mitochondrial epilepsies, and when they occur, they are usually not the main epileptic presentation (http://www.mitomap.org). For example, mutations in TSFM and RARS2, nuclear genes with a role in mitochondrial translation, were identified in patients with epilepsies but not with IS/WS. ${ }^{41,42}$ According to the two larger studies in the field, WS accounts for $14-21 \%$ (8/56 and 10/48) of the patients with epilepsy and biochemically confirmed mitochondrial respiratory chain defects. ${ }^{43,44}$ Conversely, in 13 out of the 17 children affected with IS the monitoring of body fluid metabolites suggested a defect in energy metabolism. ${ }^{45}$ Definite molecular confirmation could be completed only in a minority of patients: within the El Sabbagh's cohort, three patients had the m.8993T $>$ G variant in MT-ATP6 (ATP synthase subunit 6) while a fourth one had a variant in the nuclear SDHA gene. $^{46,47}$ The m.8993T $>\mathrm{G}$ variant is commonly found in patients with Leigh (OMIM\#256000) or NARP (neuropathy, ataxia and retinitis pigmentosa; OMIM\#551500) syndromes. Leigh syndrome is an early-onset progressive neurodegenerative disorder associated with abnormal MRI T2 signals in the basal ganglia. ${ }^{48}$ Brain MRI revealed such abnormal hypersignals in the three WS patients who shared this variant. They also showed increased CSF lactate levels, a common feature in Leigh syndrome. ${ }^{47}$ Among the 13 patients with IS reported in Shah et $a l^{45}$ a possibly causing variant was found only in two monozygotic twin sisters who had increased lactate plasma levels. They carried the m.3243A $>\mathrm{G}$ change in the tRNA ${ }^{\text {Leul }}$ gene, which is commonly associated with MELAS (mitochondrial myopathy, encephalopathy, lactate acidosis and stroke-like episodes; OMIM\#540000) syndrome. In summary, nuclear defects affecting mitochondrial protein synthesis are common within combined respiratory chain deficiencies (about one-third of patients ${ }^{49}$ ) and could result in mitochondrial epilepsies. ${ }^{40,50}$ They are, however, easily overlooked as an etiology of IS possibly because of the difficulty to correctly gauge the activity of the respiratory chain complexes, especially when no lactate and/or pyruvate increase is identified in blood and/or CSF. ${ }^{45,51}$

We show that exome sequencing is a suitable strategy to identify causative genes in familial WS and potentially IS. Exploiting this approach, we identified a novel homozygous c.1825G $>\mathrm{T}$ variant in the universally conserved elongation factor GUF1 in three siblings affected with WS. The substituted residue, that is, A609, is located within the KIIARETV stretch (defined as 'contact 2' in Connell et a ${ }^{28}$ ) of the external beta-strand of the CTD tRNA-binding moiety (Figure 2b). This beta-strand \#5 contacts on one side the CTD alpha-helix \#1 (numbering according to Connell et $a^{28}$ ) and on the other side harbors the fully conserved Q600 (539/539 alignments), a branched hydrophobic residue (364/539 I and 175/539 V) and R610 (530/539; $\mathrm{S}$ in 9/539) whose three sidechains form a tightly packed motif likely to be important for the proper recognition of the tRNA acceptor stem and D-loop. The addition of a hydroxyl group at position 609 (p.(A609S) variant) will result in a bulkier residue, which might displace the beta-sheet relative to the alpha-helix if the serine is 'looking' inside. Alternatively, as shown in Figure 2c, a S residue at this position turned toward the outside might interfere with the tRNA acceptor stem by modifying the 'contact 2' moiety. Of note, a serine residue is the only other tolerated residue at this position (42 of the 539 alignments; Supplementary Figure S4). It is, however, only found in a subgroup of bacteria such as Bactericides, Chlorobium, Clostridium, Geobacillus, Listeria, Mycoplasma, Pseudomonas and Thermoanaerobacter with tRNAs that are restricted to exactly 13 nucleotides from the beginning of the acceptor stem to the end of the D-stem. ${ }^{52}$ This stretch is always followed by an A at position 14 that pairs with the strictly conserved $\mathrm{T}$ at position 8 , a site located between the acceptor stem and the D-stem. This is in stark contrast to human mitochondrial tRNAs in which position 8 is non-constrained; any nucleotide can be positioned there and these do not necessarily form a perfect pair with the similarly non-conserved position 14 (Figure 2b). As the acceptor stem and D-stem regions have a role in binding to GUF1, we postulate that the greater variability within human mitochondrial tRNAs may result in modified interaction when the A609S mutation is present (Figure $2 \mathrm{~b}$ and $\mathrm{c}$ ).

Using complementation assays, we further demonstrate that the activity of the GUF1 ${ }^{\mathrm{A} 609 \mathrm{~S}}$ variant is modified in suboptimal conditions. GUF1 interacts genetically with MRPL36 (mitochondrial ribosomal protein L36), a gene with a crucial role in determining the rate of respiratory chain assembly. ${ }^{53}$ MRPL36 was similarly associated with LETM1 (PMID:19318571), a mitochondrial inner membrane protein, first identified in Wolf-Hirschhorn syndrome (OMIM\#194190) and associated with abnormal electrical activity in the brain. ${ }^{54,55}$ Definitive validation of the causative role of GUF1 variants in the phenotype could only be achieved through identification of more patients and engineering of animal models (a publication of a gufl mouse knockout model was recently retracted $\left.{ }^{34}\right)$. We should also consider the possibility that only few genetic changes result in a hypomorphic form of this elongation factor, a hypothesis supported by the extreme conservation of the GUF1 gene and by our inability to find more WS families with GUF1 variants until now.

In conclusion, we suggest a link between improper assembly of respiratory chain complexes and IS/WS via the identification of a variant in a fidelity factor of mitochondrial translation. 


\section{CONFLICT OF INTEREST}

The authors declare no conflict of interest.

\section{ACKNOWLEDGEMENTS}

We thank the family for its contribution. NB-K is a grantee of a young investigator grant from the University Hospital of Lyon (Hospices Civils de Lyon) and AAA is recipient of a scholarship from the Saudi Arabian National Guard Health Affairs. This work was supported by the Leenaards Foundation Prize (to AR), the Swiss National Science Foundation (to AR), the Deutsche Forschungsgemeinschaft (to JMH) and the Swiss Institute of Bioinformatics (to IX). The funders had no role in study design, data collection and analysis, decision to publish or preparation of the manuscript.

\section{AUTHOR CONTRIBUTIONS}

AAA, NBK and AR conducted the sequencing and statistical analysis. VM and JMH performed the yeast assay. NG and IX modeled the protein. DV, JdB, CR, AL, VdP, PE, MT, DS and GL phenotyped the patients. AR wrote the manuscript with contributions from DS, JMH and GL. All authors commented on and approved the manuscript.

1 Deprez L, Jansen A, De Jonghe P: Genetics of epilepsy syndromes starting in the first year of life. Neurology 2009; 72: 273-281.

2 Lux AL, Osborne JP: A proposal for case definitions and outcome measures in studies of infantile spasms and West syndrome: consensus statement of the West Delphi group. Epilepsia 2004; 45: 1416-1428.

3 Wong M, Trevathan E: Infantile spasms. Pediatr Neurol 2001; 24: 89-98.

4 Lagae L, Verhelst $\mathrm{H}$, Ceulemans B et al: Treatment and long term outcome in West syndrome: the clinical reality. A multicentre follow up study. Seizure 2010; 19 159-164

5 Pellock JM, Hrachovy R, Shinnar S et al: Infantile spasms: a U.S. consensus report. Epilepsia 2010; 51: 2175-2189.

6 Pavone P, Striano P, Falsaperla R, Pavone L, Ruggieri M: Infantile spasms syndrome, West syndrome and related phenotypes: What we know in 2013. Brain Dev 2013; 36 739-751.

7 Bahi-Buisson N, Nectoux J, Rosas-Vargas $\mathrm{H}$ et al: Key clinical features to identify girls with CDKL5 mutations. Brain 2008; 131: 2647-2661.

8 Chu-Shore CJ, Major P, Camposano S, Muzykewicz D, Thiele EA: The natural history of epilepsy in tuberous sclerosis complex. Epilepsia 2010; 51: 1236-1241.

9 Guerrini R, Moro F, Kato M et al: Expansion of the first PolyA tract of ARX causes infantile spasms and status dystonicus. Neurology 2007: 69: 427-433.

10 Mignot C, Moutard ML, Trouillard 0 et al: STXBP1-related encephalopathy presenting as infantile spasms and generalized tremor in three patients. Epilepsia 2011; 52 $1820-1827$

11 Otsuka M, Oguni H, Liang JS et al: STXBP1 mutations cause not only Ohtahara syndrome but also West syndrome-result of Japanese cohort study. Epilepsia 2010; 51: 2449-2452

12 Weaving LS, Christodoulou J, Williamson SL et al: Mutations of CDKL 5 cause a severe neurodevelopmental disorder with infantile spasms and mental retardation. Am J Hum Genet 2004; 75: 1079-1093.

13 Boutry-Kryza N, Ville D, Labalme A et al: Complex mosaic CDKL5 deletion with two distinct mutant alleles in a 4-year-old girl. Am J Med Genet A 2014; 164A 2025-2028.

14 Paciorkowski AR, Thio LL, Dobyns WB: Genetic and biologic classification of infantile spasms. Pediatr Neurol 2011; 45: 355-367.

15 Osborne JP, Lux AL, Edwards SW et al: The underlying etiology of infantile spasms (West syndrome): information from the United Kingdom Infantile Spasms Study (UKISS) on contemporary causes and their classification. Epilepsia 2010; 51: 2168-2174.

16 Partikian A, Mitchell WG: Neurodevelopmental and epilepsy outcomes in a North American cohort of patients with infantile spasms. J Child Neurol 2010; 25 : 423-428.

17 Michaud JL, Lachance M, Hamdan FF et al: The genetic landscape of infantile spasms. Hum Mol Genet 2014; 23: 4846-4858.

18 Epi KC, Epilepsy Phenome/Genome P, Allen AS et al: De novo mutations in epileptic encephalopathies. Nature 2013; 501: 217-221.

$19 \mathrm{Li} \mathrm{H}$, Durbin R: Fast and accurate short read alignment with Burrows-Wheeler transform. Bioinformatics 2009; 25: 1754-1760.

20 DePristo MA, Banks E, Poplin R et al: A framework for variation discovery and genotyping using next-generation DNA sequencing data. Nat Genet 2011; 43: 491-498.
21 McKenna A, Hanna M, Banks E et al: The Genome Analysis Toolkit: a MapReduce framework for analyzing next-generation DNA sequencing data. Genome Res 2010; 20 1297-1303.

22 Danecek P, Auton A, Abecasis G et al: The variant call format and VCFtools. Bioinformatics 2011; 27: 2156-2158.

23 Bauerschmitt H, Funes S, Herrmann JM: The membrane-bound GTPase Guf1 promotes mitochondrial protein synthesis under suboptimal conditions. J Biol Chem 2008; 283 17139-17146.

24 Lemaire C, Guibet-Grandmougin F, Angles D, Dujardin G, Bonnefoy N: A yeast mitochondrial membrane methyltransferase-like protein can compensate for oxal mutations. J Biol Chem 2004; 279: 47464-47472.

25 Szyrach G, Ott M, Bonnefoy N, Neupert W, Herrmann JM: Ribosome binding to the Oxa1 complex facilitates co-translational protein insertion in mitochondria. EMBO 2003; 22: 6448-6457.

26 Ng PC, Henikoff S: Predicting deleterious amino acid substitutions. Genome Res 2001; 11: 863-874.

27 Evans RN, Blaha G, Bailey S, Steitz TA: The structure of LepA, the ribosomal back translocase. Proc Natl Acad Sci USA 2008; 105: 4673-4678.

28 Connell SR, Topf M, Qin $Y$ et al: A new tRNA intermediate revealed on the ribosome during EF4-mediated back-translocation. Nat Struct Mol Biol 2008; 15 910-915.

29 Guex N, Peitsch MC: SWISS-MODEL and the Swiss-PdbViewer: an environment for comparative protein modeling. Electrophoresis 1997; 18: 2714-2723.

30 Qin Y, Polacek N, Vesper 0 et al: The highly conserved LepA is a ribosomal elongation factor that back-translocates the ribosome. Cell 2006; 127: 721-733.

31 Zhang D, Qin Y: The paradox of elongation factor 4: highly conserved, yet of no physiological significance? Biochem J 2013; 452: 173-181.

32 Shoji S, Janssen BD, Hayes CS, Fredrick K: Translation factor LepA contributes to tellurite resistance in Escherichia coli but plays no apparent role in the fidelity of protein synthesis. Biochimie 2010; 92: 157-163.

33 Bijlsma JJ, Lie ALM, Nootenboom IC, Vandenbroucke-Grauls CM, Kusters JG: Identification of loci essential for the growth of Helicobacter pylori under acidic conditions. J Infect Dis 2000; 182: 1566-1569.

34 Piao YR, Jin ZH: Loss of Guf1 impairs sperm mitochondrial function and leads to male infertility. Biol Reprod 2015; 92: 117.

35 Yang F, Gao Y, Li Z et al: Mitochondrial EF4 links respiratory dysfunction and cytoplasmic translation in Caenorhabditis elegans. Biochim Biophys Acta 2014; 1837 1674-1683

36 Pech M, Karim Z, Yamamoto H, Kitakawa M, Qin Y, Nierhaus KH: Elongation factor 4 (EF4/LepA) accelerates protein synthesis at increased Mg2+ concentrations. Proc Nat Acad Sci USA 2011; 108: 3199-3203.

37 Liu H, Chen C, Zhang H, Kaur J, Goldman YE, Cooperman BS: The conserved protein EF4 (LepA) modulates the elongation cycle of protein synthesis. Proc Natl Acad Sci USA 2011; 108: 16223-16228.

38 Debray FG, Lambert M, Chevalier I et al: Long-term outcome and clinical spectrum of 73 pediatric patients with mitochondrial diseases. Pediatrics 2007; 119: $722-733$.

39 Khurana D, Salganicoff L, Melvin J et al: Epilepsy and respiratory chain defects in children with mitochondrial encephalopathies. Epilepsia 2008; 49: 1972.

40 Rahman S: Mitochondrial disease and epilepsy. Dev Med Child Neurol 2012; 54 397-406

41 Smeitink JA, Elpeleg O, Antonicka $\mathrm{H}$ et al: Distinct clinical phenotypes associated with a mutation in the mitochondrial translation elongation factor EFTs. Am J Hum Genet 2006; 79: 869-877.

42 Edvardson S, Shaag A, Kolesnikova 0 et al: Deleterious mutation in the mitochondrial arginyl-transfer RNA synthetase gene is associated with pontocerebellar hypoplasia. Am J Hum Genet 2007; 81: 857-862.

43 Lee YM, Kang HC, Lee JS et al: Mitochondrial respiratory chain defects: underlying etiology in various epileptic conditions. Epilepsia 2008; 49: 685-690.

$44 \mathrm{El}$ Sabbagh S, Lebre AS, Bahi-Buisson N et al: Epileptic phenotypes in children with respiratory chain disorders. Epilepsia 2010; 51: 1225-1235.

45 Shah NS, Mitchell WG, Boles RG: Mitochondrial disorders: a potentially underrecognized etiology of infantile spasms. J Child Neurol 2002; 17: 369-372.

46 Horvath R, Abicht A, Holinski-Feder E et al: Leigh syndrome caused by mutations in the flavoprotein $(\mathrm{Fp})$ subunit of succinate dehydrogenase (SDHA). J Neurol Neurosurg Psychiatry 2006; 77: 74-76.

47 Desguerre I, Pinton F, Nabbout R et al: Infantile spasms with basal ganglia MR hypersignal may reveal mitochondrial disorder due to T8993G MT DNA mutation. Neuropediatrics 2003; 34: 265-269.

48 Lee HF, Tsai CR, Chi CS, Lee HJ, Chen CC: Leigh syndrome: clinical and neuroimaging follow-up. Pediatr Neurol 2009; 40: 88-93.

49 Kemp JP, Smith PM, Pyle A et al: Nuclear factors involved in mitochondrial translation cause a subgroup of combined respiratory chain deficiency. Brain 2011; 134 $183-195$

50 Desguerre I, Hully M, Rio M, Nabbout R: Mitochondrial disorders and epilepsy. Rev Neurol 2014; 170: 375-380.

51 Desguerre I, Nabbout R, Dulac 0: The management of infantile spasms. Arch Dis Child 2008; 93: 462-463.

52 Juhling F, Morl M, Hartmann RK, Sprinzl M, Stadler PF, Putz J: tRNAdb 2009: compilation of tRNA sequences and tRNA genes. Nucleic Acids Res 2009; 37: D159-D162.

53 Prestele M, Vogel F, Reichert AS, Herrmann JM, Ott M: Mrpl36 is important for generation of assembly competent proteins during mitochondrial translation. $\mathrm{Mol} \mathrm{BiO}$ Cell 2009; 20: 2615-2625. 
54 Jiang D, Zhao L, Clish CB, Clapham DE: Letm1, the mitochondrial Ca2+/H+ antiporter, is essential for normal glucose metabolism and alters brain function in Wolf-Hirschhorn syndrome. Proc Natl Acad Sci USA 2013; 110: E2249-E2254.
55 Schlickum S, Moghekar A, Simpson JC et al: LETM1, a gene deleted in WolfHirschhorn syndrome, encodes an evolutionarily conserved mitochondrial protein. Genomics 2004; 83: 254-261.

Supplementary Information accompanies this paper on European Journal of Human Genetics website (http://www.nature.com/ejhg) 